CUAD. CONTAB. / BOCOTÁ, COLOMBIA, 15 (39): 883-895 / NÚMERO ESPECIAL 2014 / 883

\title{
Determinantes de costos de auditoría - non audit fee
}

doi: 10.11144/Javeriana.cc15-39.dcan

\section{Arquimedes de Jesus Moraes}

Vila Velha Universidad, Campus Business School, bulevar comercial de Vila Velha, Espírito Santo, Brasil. Licenciatura en Contabilidad, Universidad de Vila Velha, UVV. Especialista en contabilidad y auditoría forense, Universidad Cândido Mendes, UCAM. Maestría en contabilidad, Fundación Instituto Capixaba de Investigación en Contabilidad, Economía y Finanzas, FUCAPE. Auditor independiente y consultor de empresas. Socio director de la CEHR Consultores y Asociados, se dedica en la prestación de servicios de consultoría de organización fiscal y con un enfoque en las micro y pequeñas empresas, proveedores de servicios, industria y las instituciones sin fines de lucro. Correos electrónicos: Arquimedes.moraes@uvv.br, Arquimedes.moraes@gmail.com

\section{Eduardo José Pinheiro}

Vila Velha Universidad, Campus Business School, bulevard comercial de Vila Velha, Espírito Santo, Brasil. Licenciado en contabilidad, Universidad Federal de Espírito Santo. Especialista en planeamiento de la educación, Universo. Especialista en Contraloría, Sedes UVV. Maestro en educación, Instituto Pedagógico Latinoamericano y Caribeño, Cuba, 2003, con grado revalidado en la Universidad Federal de Pernambuco en 2008. Profesor y coordinador de la Universidad Vila Velha. Educador de Contabilidad desde
1990 y desde 1993 actúa en el mercado como experto contable, auditor, profesor y cursos de contabilidad. En la Universidad de Vila Velha, coordina TCC, actividades de enriquecimiento, las pasantías y la parte pedagógica. Profesor de introducción a la contabilidad, estructura de los estados I y II, control interno, TCC I y II, contabilidad y especialización en auditoría, entre otros.

Correo electrónico: edupinheiro@uvv.br edujpinheiro@gmail.com

\section{Alexsandro Ronchi Negrelli}

Vila Velha Universidad, Campus Business School, bulevar comercial de Vila Velha, Espírito Santo, Brasil. Estudiante de maestría en educación internacional, Anne Sullivan University, ASU. Estudiante de maestría en Contabilidad, FUCAPE. Executive MBA en contabilidad y finanzas, FUCAPE. Licenciatura en ciencias en contabilidad, UVV. Técnico en contabilidad, ESG Prof. Geoffrey Schneider. Profesor de contabilidad, curso de coordinación universitaria, asistente Vila Velha, UVV. Profesor de análisis de estados financieros, estructura de estados financieros I y temas especiales en Contabilidad. Opera en el mercado, en empresas de negocios de comercio exterior desde 1992, donde asesora en áreas contables y fiscales.

Correos electrónicos: Alexsandro.negrelli@uvv.br arnegrelli@msn.com 
Resumen El objetivo de esta investigación es identificar determinantes de los costos de auditoría (non audit FEE) de las empresas brasileñas. La muestra contempló informaciones de las empresas en la BM\&FBOVESPA, que resulta de la fusión entre la São Paulo Stock Exchange (Bovespa) y la Brazilian Mercantile and Futures Exchange (BM\&F), entre 2009 y 2012. Los resultados indican que el tamaño de la empresa auditada en el final del año fiscal está positivamente relacionado con los gastos de consultoría; si la empresa de auditoría es una Big 4, los honorarios de consultoría no poseen una relación significativa. En contrapartida, los clientes de menor tamaño tendrán costes menores de honorarios de consultoría.

Palabra clave honorarios de consultoría; Big 4; calidad de auditoría

\section{Código JEL M 41}

\section{Determinants of Audit Fees - Non Audit Fee}

Abstract The objective of this research is to identify the determinants of the audit fees (non audit FEE) of Brazilian companies. The sample considered information from the companies in the BM\&FBOVESPA, resulting from the fusion between the Sao Paulo Stock Exchange (Bovespa) and the Brazilian Mercantile and Futures Exchange (BM\&F), between 2009 and 2012. The results show that the size of the audited company at the end of the fiscal year is related positively with the consulting expenses; if the auditing company is part of the Big 4, the consulting fees do not show a significant relationship. On the other hand, the smaller clients have lower costs related to consulting fees.

Keywords consulting fees; Big 4; auditing quality

\section{Determinantes de custos de auditoria - non audit fee}

Resumo O objetivo desta pesquisa é identificar determinantes dos custos de auditoria (non audit FEE) das empresas brasileiras. A amostra incluiu informações das empresas na BM\&FBOVESPA, que resulta da fusão entre a São Paulo Stock Exchange (Bovespa) e a Brazilian Mercantile and Futures Exchange (BM\&F), entre 2009 e 2012. Os resultados indicam que o comprimento da empresa auditada no final do ano fiscal é relacionado positivamente com as despesas de consultoria; caso da empresa de auditoria ser uma Big 4 , os honorários de consultoria não possuem relacionamento significativo. Em contrapartida, os clientes de menor comprimento terão custos menores de honorários de consultoria.

Palavras-chave honorários de consultoria; Big 4; qualidade de auditoria

\section{Introducción}

El auditor actúa en empresas con necesidades diferentes en relación con su tamaño e importancia económica. Debido a este hecho, el presente estudio investiga la existencia de una relación entre el tamaño de las empresas y el valor de las comisiones de asesoría. El auditor independiente de la contabilidad es un agente de gran importancia en el mercado financiero en general, porque este profesional actúa como intermediario entre los preparadores de los estados financieros y sus usuarios (Hanlon, Krishnan \& Mills, 2012).

Estudiosos como William R. Kinney y Robert Libby (2002), John Daniel Eshleman y Peng Guo (2013) investigaron los efectos que los honorarios de auditoría pueden tener en la calidad de los servicios prestados y el costobeneficio. A partir de este hecho, el objetivo de este artículo es verificar la existencia de una relación entre el valor de los honorarios de asesoría y el tamaño de las empresas brasileñas auditadas que cotizan en la BM\&FBovespa.

La encuesta que da origen a este texto confirmó que el auditor considera el tamaño de la empresa cliente como parámetro para fija los honorarios de su consultoría. La relación inversa entre la variable de interacción de audi- 
tor, las Big $4^{1}$ y los honorarios de consultoría se considera como relevante pero no es significativa en términos estadísticos; esto significa que las cuatro firmas contables más grandes del mundo no cobran las tarifas más altas a las empresas que contratan sus servicios.

El propósito de esta investigación es analizar la relación entre el tamaño de la empresa y el valor de los honorarios de asesoría.

\section{Metodología}

Se utiliza el modelo desarrollado por Parveen P. Gupta, Gopal V. Krishnan y Wei Yu (2012). Este modelo solo se ha modificado de acuerdo con la disponibilidad de datos y adaptado a la realidad brasileña.

Vale la pena agregar que se llevó a cabo el análisis de la influencia del tamaño de la empresa en el valor comprobado de los honorarios de asesoría mediante modelos de regresión.

\subsection{Base de datos de muestra}

La muestra se compone de 300 empresas públicas del mercado BM\&FBOVESPA en el período 2009-2012. La definición de este período se debe a la disponibilidad de datos de los honorarios de auditoría de 2009 y la limitación de la base existente en 2012 .

Los datos financieros fueron extraídos de los estados financieros estandarizados disponibles en las páginas web de la BM\&FBovespa y la base de datos Economática. La muestra final consta de 300 empresas; las exclusiones obedecen a la falta de datos disponibles, en última

1 Las Big 4 son PwC (PricewaterhouseCoopers), Deloitte Touche Tohmatsu, Ernst \& Young y KPMG. instancia, quedaron 832 observaciones al año por empresa.

El período de estudio comprende de 2009 a 2012. La fecha de inicio se da en el formulario de referencia obligatoria (declaración con los elementos de los honorarios de auditoría que han sido proporcionados por la Instrucción CVM 480 de 7 de diciembre de 2009) y la fecha de finalización está vinculada a la disponibilidad de los datos básicos de los honorarios de auditoría.

\subsection{Variables empíricas}

\subsubsection{Remuneración de los auditores - variable dependiente}

Las tasas de consultoría son la variable dependiente, pues el objetivo principal de la investigación es investigar si hay una relación entre los honorarios satisfechos al auditor externo y el tamaño de la empresa auditada.

Como se evidencia en la literatura, los riesgos de gestión de los resultados de la auditoría están vinculados a las tasas cobradas y a la ganancia o pérdida de las empresas.

De este modo, el operador trata de compensar los riesgos asumidos, los litigios y la pérdida de reputación a los que está sometido (Hanlon, Krishnan \& Mills, 2012).

\subsubsection{Tamaño de la firma - interés variable}

La variable de interés, que se explica en esta investigación, es la misma que se expone en la obra de Jere R. Francis (1984): el logaritmo natural de los activos totales de las empresas al final del año fiscal. 
En esta variable, Zoe-Vonna Palmrose (1986) hace hincapié en que la importancia y el tamaño de la empresa son los principales factores que explican los servicios de fijación de precios de auditoría, y nos advierte que este resultado solo puede ser natural, debido al hecho de que será necesario un mayor esfuerzo de la firma de auditoría para analizar la empresa. Por cierto, vale la pena agregar que Francis (1984) relaciona los honorarios de auditoría con los activos totales de las empresas auditadas.

\subsubsection{Variables de control}

Podría controlar el efecto del tamaño de las empresas de auditoría, se añadió el grupo de las Big 4. Esta variable ficticia - firma de observación/año-corresponde al hecho de que la empresa auditora es una de las cuatro (4) principales: PricewaterhouseCoopers Deloitte Touche Tohmatsu, Ernst \& Young, y KPMG.

Para incluir las consecuencias de la situación financiera presentes en cada observación empresa/año, se agregó la variable retorno sobre activos (Return on Assets, ROA) como una medida de rendimiento que se encuentra dividiendo la utilidad neta por los activos totales y muestra el porcentaje de los ingresos generados por el activo.

\subsection{Modelo de regresión}

Una vez señaladas las variables utilizadas, ahora tenemos el modelo del estudio que es una adaptación del modelo de Parveen P. Gupta, Gopal V. Krishnan y Wei Yu (2012):

$$
\text { NONAUDIT_FEES } \left.S_{i t}=\beta_{0}+\beta_{1} \text { LTA }\right)+\beta_{2}
$$

$\mathrm{BIG} 4+\beta_{3} \mathrm{MTB}_{\mathrm{it}}+\beta_{4} \mathrm{LEV}_{\mathrm{it}}+\beta_{5} \mathrm{ROA}_{\mathrm{it}}+\beta_{6}$ ARIVN $_{\text {it }}+\beta_{7}$ TENURE $_{\text {it }}+\beta_{8}$ REPLAG $_{i t}+\beta_{9}$
SEGMENT $_{\text {it }}+\beta_{10}$ LAGE $_{\text {it }}+\beta_{11}$ SGROWTH $_{\text {it }}+$ $\beta_{12}$ RESTATE $_{\mathrm{it}}+\varepsilon_{\mathrm{it}}$

Donde:

NONAUDIT_FEES: honorarios de consultas recibidas por la sociedad de auditoría

LTAT: logaritmo natural de los activos totales al final del año fiscal

BIG4t: variable ficticia con valor 1 , si la firma de auditoría pertenece a las Big 4

MTBT: índice market-to-book de la empresa, definido como el valor de mercado de las acciones, mediante la división del valor contable del patrimonio neto

LEVT: activos totales de la empresa menos el valor neto dividido por el total de activos

Roat: rentabilidad sobre activos totales

ARINVt: sumatoria de las cuentas por cobrar y el inventario de la empresa dividida por el total de sus activos

TENUREt: mandato en tiempo del auditor REPLAGt: número de días entre el final del año fiscal y el anuncio de los resultados

SEGMENTt: número de segmentos de negocio de la empresa

Laget: iniciar sesión natural de edad de la empresa

SGROWTHt: tasa de crecimiento de las ventas con respecto al año fiscal anterior

RESTATE: variable de indicador que es igual a 1 si la empresa cuenta con una corrección del balance, y 0 , en caso contrario

\section{Marco teórico}

\subsection{Auditoría externa}

La auditoría externa tiene por objeto confirmar o no, por medio de una opinión, los criterios 
adoptados por la empresa en la preparación de las cuentas y los registros de los hechos económicos y su difusión por medio de los informes financieros que cumplen las normas de contabilidad. En Brasil, la Ley de Sociedades (Ley 6.404/76, art. 177) determina que los informes financieros de las empresas públicas deben estar sujetos a revisión por las empresas de auditoría independientes y tal determinación se extendió a las grandes empresas por la Ley 11.638/07.

De acuerdo con el Comité de Pronunciamientos Contables (Comitê de Pronunciamentos Contábeis, CPC), el objetivo de los informes financieros es suministrar información acerca de las transacciones y otros sucesos pasados útiles para los usuarios en la toma de decisiones económicas. En cuanto a los atributos, esta información es útil porque tiene cuatro características cualitativas: comprensibilidad, relevancia, fiabilidad y comparabilidad. Específicamente, la información es fiable si está libre de error o riesgo relevante y representa adecuadamente lo que propone (CPC, 2008, artículos 22, 24 a 32, pp. 10-12).

Tanto en la preparación de los informes financieros como en el trabajo desarrollado, la independencia de los auditores es el tema principal y fundamental para la calidad de la información divulgada y la credibilidad de la función del auditor. La independencia del auditor, por tanto, es la base para dos de las características más importantes de la profesión de auditor: credibilidad y confianza. Esto significa que todos los agentes implicados en la información contable derivada de la prestación de servicios de auditoría (la contratante, el contratista y el usuario) tendrán una mayor fiabilidad de la información (Formigoni, Antunes, Leite \& Paulo, 2008).

\subsection{Independencia de la auditoría}

La resolución 1034/05 del Consejo Federal de Contabilidad (Conselho Federal de Contabilidade, CFC) aprueba la NBC P 1.2 - Normas Profesionales del Auditor independiente para establecer las condiciones y procedimientos para el cumplimiento de los requisitos de independencia profesional en auditoría. En la conceptualización de la independencia, se expresa su importancia para la calidad del trabajo de auditoría: la independencia es una condición fundamental y obvia para el ejercicio de toda auditoría independiente.

La independencia se entiende como el estado en el que las obligaciones o intereses de la entidad de auditoría están libres de las obligaciones o intereses de las entidades auditadas, lo que les permite prestar sus servicios con objetividad. En pocas palabras, es la capacidad que la entidad de auditoría tiene para juzgar y actuar con integridad y objetividad al presentar informes de habilitación y una asesoría imparcial sobre la entidad auditada, con destino a los accionistas, socios comerciales, miembros y todos los que puedan estar relacionados con su trabajo (Formigoni, Antunes, Leite \& Paulo, 2008).

\subsection{Calidad de las auditorías en Brasil}

La independencia es un atributo importante en la calidad de la auditoría, considerando el papel desempeñado por el auditor en el contexto de la información contable (Braunbeck, 2010). 
En un mercado competitivo con una demanda de diferenciación de servicios, una firma contable cobrará más dinero por ofrecer servicios de alta calidad (Francis, 1984). Por tanto, los honorarios de auditoría pueden ser utilizados para analizar la calidad de las auditorías.

Linda Elizabeth DeAngelo (1981) y Ross L. Watts y Jerold L. Zimmerman (1986) señalan que no es suficiente que el auditor sea capaz de detectar errores y/o fraudes (competencia técnica), sino que también debe tener la voluntad de informar de manera apropiada (independencia).

Según David F. Larcker y Scott Richardson (2004), en teoría, si el auditor tiene dependencia financiera de su cliente en particular, o si una parte relevante de sus ingresos depende de este, la violación de la independencia es más probable, dado que la firma de auditoría tendrá más dificultad en señalar los errores en los informes financieros por el riesgo que esto significa en sus beneficios futuros.

Es fundamental señalar que para la auditoría externa alcanzar su objetivo de reducir las asimetrías de información debe haber independencia (Hallak \& Silva, 2012).

Para Richard M. Frankel, Marilyn F. Johnson y Karen K. Nelson (2002), uno de los principales problemas para la independencia es la venta de servicios de consultoría (o fee, no de auditoría, como se llama aquí) por las empresas de auditoría.

\subsection{Determinantes de los honorarios de auditoría}

Los estudios sobre la posible relación entre el gobierno corporativo de las empresas y la cali- dad del costo de los servicios de auditoría independientes son relativamente recientes. Como se ve en el marco teórico, la literatura sobre el tema es bastante reciente, ya que los primeros estudios sobre los honorarios de auditoría fueron escritos en los años 80.

Jere R. Francis (1984), uno los estudiosos del tema en los años ochenta, sostiene que en un mercado competitivo en el que hay una demanda de diferenciación de servicios, una firma contable requerirá un valor más alto para ofrecer mejores servicios. Por tanto, igual que Rodrigo Telles Pires Hallak y Andre Luiz Carvalhal da Silva (2012), creemos que el análisis de la calidad de las auditorías se puede realizar a partir de estos honorarios.

Los investigadores Don A. Moore, Philip E. Tetlock, Lloyd Tanlu y Max H. Bazerman (2006) señalan en su estudio la falta de independencia de los auditores externos estadounidenses como uno de los principales factores de los numerosos escándalos financieros en los años noventa y principios del siglo XXI. Los estudiosos presentan tres posibles amenazas a la independencia del auditor: (i) la contratación de los auditores por los directivos que serán auditados, (ii) los auditores toman posiciones en las empresas clientes y (iii) la prestación de servicios adicionales (distintos de auditoría).

Moore, Tetlock, Tanlu y Bazerman (2006) también señalan que los servicios distintos de la auditoría pueden comprometer la independencia del servicio de dos maneras: (i) el riesgo de la auditoría a ser económicamente dependiente del cliente y la preocupación por la pérdida de ingresos, lo que lleva a "prevenir" un dictamen negativo y (ii) la prestación de ser- 
vicios de consultoría puede poner al auditor en una posición similar a la de los administradores, lo que hará peligrar su juicio.

En resumen, los honorarios de los auditores se pueden utilizar para comprobar la calidad del servicio proporcionado a las empresas (Hallak \& Silva, 2012). Por tanto, es evidente que no es suficiente que el auditor tenga experiencia; es importante, además, que el auditor sea independiente de la empresa que contrató sus servicios (DeAngelo, 1981; Watts \& Zimmerman, 1986).

Como se puede ver, hace algún tiempo que los investigadores muestran curiosidad por los efectos que los pagos pueden tener en la calidad y eficacia de los servicios de auditoría prestados.

Por tanto, hay dos formas que se describen en esta afirmación para observar la literatura (Eshleman \& Guo, 2013). En primer lugar, William R. Kinney y Robert Libby (2002) señalan que los pagos de auditoría más grandes (los llamados anormales) son muestra de las rentas ilícitas o futuros, que serán parte de la cantidad recaudada por el auditor. Así tenemos la siguiente situación: los honorarios de auditoría más altos (en relación con el número de horas requeridas en el trabajo) puede hacer que el auditor pierda su independencia, lo que da espacio a que el contratista ponga en duda al auditor (Eshleman \& Guo, 2013).La segunda pregunta que se hacen los investigadores Eshleman y Guo (2013), en relación con el control de la calidad de los servicios de auditoría realizados como alerta, obedece al hecho de que los pagos de auditoría más grandes representan un mayor compromiso y, por tanto, el desempeño de los auditores y el resultado de esta variable "suma” será una auditoría adecuada porque el auditor, debido a su alto sueldo se esforzará más para obtener mejores resultados. Los honorarios bajos de auditoría llevan a un trabajo descuidado y mal hecho realizado por el auditor. Por tanto, los bajos salarios estarían relacionados con una menor calidad de la auditoría.

\subsection{Honorarios de auditoría}

El primer modelo de auditoría, desarrollado por Dan A. Simunic (1980), mide las variables que determinan el monto de los honorarios de auditoría. Simunic (1980) considera varios aspectos de las empresas auditadas, como su tamaño, la complejidad de sus actividades, su situación financiera, entre otros. Este primer modelo fue desarrollado con la intención de analizar la situación de monopolio de la que se acusó a las ocho firmas de auditoría más grandes de la época (Arthur Andersen; Arthur Young \& Co.; Coopers \& Lybrand; Ernst \& Whinney; Deloitte Haskins \& Sells, y Deloitte \& Co.; Peat Marwick Mitchell; Price Waterhouse; y Touche Ross).

La investigación actual puso de relieve el análisis de la existencia de una relación entre los honorarios de auditoría y gestión de los ingresos, o incluso la calidad de los beneficios generados. Jean C. Bedard y Karla Johnstone (2004) informaron sobre las empresas que han demostrado resultados de la gestión del riesgo pertinentes. Los planes de actuación de los auditores tuvieron que ser revisados y, por tanto, se promovió un aumento en el monto de los pagos.

Gopal V. Krishnan y Gnanakumar Visvanathan (2009) sostienen que el riesgo de gestión 
de resultados es relevante para los auditores, debido al hecho de que aumenta el riesgo de reediciones y, posteriormente, el riesgo de auditoría en su conjunto.

\section{Análisis de los resultados}

En este capítulo, se discuten los resultados obtenidos de las pruebas empíricas y se presentan las estadísticas descriptivas de esta investigación.

\subsection{Estadística descriptiva y análisis de correlación}

En este tema se presentan las estadísticas descriptivas y el análisis de correlación de Spearman $(\rho)$.

Del análisis de la tabla 1 , se destacan algunos detalles de la muestra que son relevantes para la correcta interpretación de los resultados. En primer lugar, podemos ver que la firma de estadísticas / año fueron auditados por una firma de auditoria Big 4.

El promedio de la variable Big 4 es $82.51 \%$; esta variable es binaria, es decir, muestra la ocurrencia o no de algún hecho, y se puede entender que su media representa las observaciones empresa/año en las cuales el auditor era una Big 4.

En segundo lugar, en los índices se observa que los hallazgos empresa/año tardaron un promedio de 78 días para ser liberados al público.

La tabla 2 presenta la correlación de Spearman $(\rho)$ y tiene la intención de identificar los posibles efectos de multicolinealidad entre las variables del modelo. Ambas variables - Log LTA y BIG 4- tienen correlación moderada y débil con la variable NONAUD FEES, del mismo modo que LTA lo tiene con BIG 4 y MTB.

\subsection{Análisis de regresión}

Se presentan y analizan los resultados de las regresiones en este tema. Los resultados se obtuvieron desde el análisis de regresión lineal agrupado. Por tanto, la ecuación se ensayó para evaluar la influencia del tamaño de la empresa auditada en los honorarios de una consultoría determinados por el tamaño de la empresa (Big 4) y el rendimiento sobre los activos (ROA), entre otros, como se puede ver en la tabla 3.

Los resultados de la tabla 4 demuestran que el modelo desarrollado tiene un valor predictivo del $17 \%$, aproximadamente. Las estimaciones de la $\mathrm{R}^{2}$ ajustada son de $18 \%$ o 0,178497 y tienen valor $\mathrm{R}^{2}$ de 0.178497 .

En los resultados de la tabla 3, hay que señalar que las variables BIG4, LTA, ARINV y REPLAG han representado una estadística $t$ significativa. Al analizar los coeficientes, podemos destacar una relación positiva entre la LTA y los honorarios de consultoría percibidos por la firma de auditoría, así como el índice de marketto-book, ROA, la posesión del auditor en años, SEGMENT y SGROWTH. Los datos con signos negativos en sus coeficientes fueron: BIG4, LEV, ARINV, REPLAG y SGROWTH. Esto significa, por tanto, que hay una relación inversa con los honorarios de auditoría.

También en relación con los resultados de la tabla 3, se destaca la variable más importante: LTA - logaritmo natural de los activos totales al final del año fiscal. Esta variable fue muy significativa para indicar que el auditor tiende a requerir una mayor compensación cuando trabaja en empresas con mayores activos. Esto sugiere que una gran cantidad de activos es sinónimo de complejidad, porque hay una mayor dificultad en la realización de la obra. 


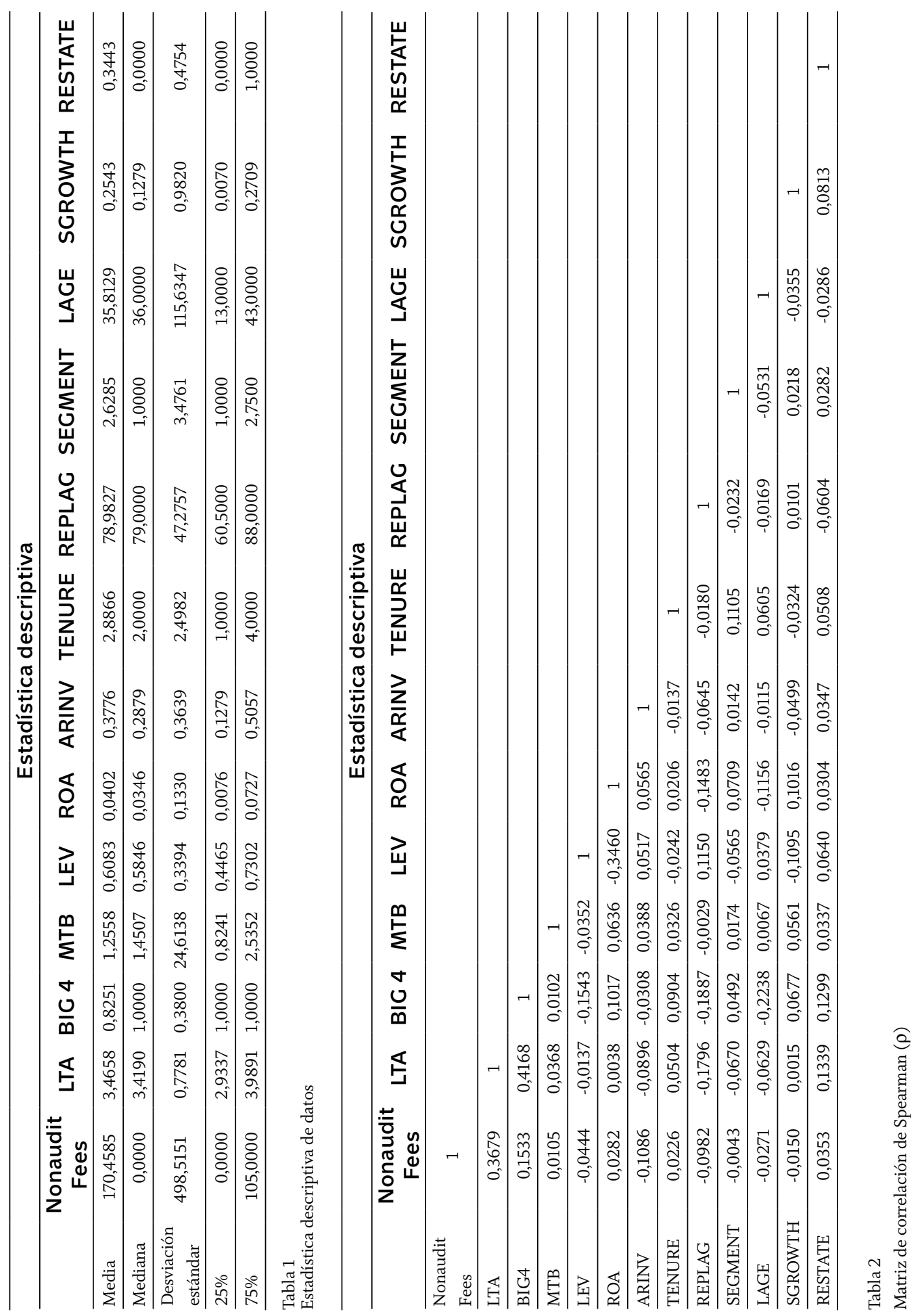




\begin{tabular}{|c|c|c|c|c|}
\hline & Coeficientes & Stat-t & Valor-P & Error estándar \\
\hline Intersección & $-800,4567$ & $-6,4773$ & 0,0000 & 123,5781 \\
\hline LTA & 325,1282 & 11,3910 & 0,0000 & 28,5426 \\
\hline BIG4 & $-42,4784$ & $-0,7265$ & 0,4677 & 58,4673 \\
\hline MTB & 1,9205 & 0,5374 & 0,5911 & 3,5736 \\
\hline LEV & $-6,5334$ & $-0,1121$ & 0,9107 & 58,2642 \\
\hline ROA & 29,7984 & 0,1999 & 0,8416 & 149,0453 \\
\hline ARINV & $-135,5096$ & $-2,7022$ & 0,0070 & 50,1472 \\
\hline TENURE & 3,1252 & 0,4259 & 0,6703 & 7,3383 \\
\hline REPLAG & $-0,6777$ & $-1,7879$ & 0,0742 & 0,3790 \\
\hline SEGMENT & 1,2701 & 0,2317 & 0,8168 & 5,4816 \\
\hline LAGE & 0,9849 & 0,8949 & 0,3711 & 1,1006 \\
\hline SGROWTH & $-8,2201$ & $-0,4381$ & 0,6614 & 18,7621 \\
\hline RESTATE & 17,5955 & 0,4514 & 0,6518 & 38,9809 \\
\hline
\end{tabular}

Tabla 3

Resultados de regresión

\begin{tabular}{lc}
\hline \multicolumn{2}{c}{ Regresión estadística } \\
\hline R2 & 0,178497 \\
\hline R2 ajustada & 0,16646 \\
\hline Error estándar & 521,8997 \\
\hline F estadística & 15 \\
\hline Observaciones & 832 \\
\hline
\end{tabular}

Tabla 4

Regresión estadística

Se añadió la variable BIG4 para capturar el efecto esperado de que los servicios de un auditor con una carga más grande, costarían más que los servicios de los auditores no BIG4, pero el coeficiente no tenía ningún efecto, lo que significaría su relación inversa con las comisiones de asesoría; sin embargo, el P-valor no fue significativo.

La salud financiera de la entidad auditada es relevante para el auditor; la variable ROA, que mide el desempeño financiero, presentó un coeficiente positivo; sin embargo, no significativo.
Por tanto, no podemos decir que a mejor desempeño financiero de la empresa auditada, mayores honorarios percibe la consultora.

La percepción que se tiene de la variable ARINV es que cuando los auditores tienen cuentas por cobrar y artículos en el inventario, entienden que tienen que hacer más pruebas, circularización e inventario. Estos factores se entienden como aquello que incrementa el costo de la auditoría. En este trabajo, el coeficiente fue negativo y el valor $P$ fue significativo. Este índice muestra que las cuentas por cobrar y los artícu- 
los del inventario en la empresa no imponen mayores costos de los honorarios de consultoría.

\section{Conclusión}

Esta investigación tuvo como objetivo investigar la existencia de relación entre los honorarios de consultoría y el tamaño de las empresas auditadas. El modelo de regresión utilizado fue una adaptación de los modelos de Parveen P. Gupta, Gopal V. Krishnan y Wei Yu (2012).

Para responder la pregunta de investigación se utilizó una muestra recogida por la base de datos Economática.

Se estudiaron 300 compañías públicas que cotizan en la BM\&FBOVESPA, en el período 2009-2012.

Los resultados demuestran estadísticamente que el auditor externo considera el tamaño de las empresas auditadas en la fijación de los honorarios de asesoría.

Por tanto, los auditores requieren una mayor compensación, es decir, las grandes empresas pagan un sueldo considerable a los auditores en concepto de honorarios de consultoría - nonaudit-fee. El principal aporte de este estudio, por tanto, es la capacidad de ver que el tamaño de la empresa cliente se relaciona positivamente con los honorarios de consultoría.

Esta investigación muestra, en última instancia, la percepción de riesgo del auditor y que este factor se refleja en su remuneración.

Los resultados implican también a los miembros del Comité de Auditoría y la alta dirección de empresas en la contratación de los auditores y la negociación de los honorarios de auditoría.
Una limitación de este estudio es que el análisis se concentró solo en las grandes empresas. Por tanto, no se evaluará la totalidad del universo de las empresas auditadas que también son empresas cerradas y/o pequeñas empresas.

Por último, señalamos que hay oportunidades para una mayor investigación, por ejemplo, la determinación de otros tipos de gastos de auditoría, a saber: i) el porcentaje de ventas en el exterior debido a las ventas totales (FORGN); ii) si la empresa auditada participa en un plan de pensiones (PENSIONES), iii) si la empresa auditada tiene debilidades en su control interno (ICW).

\section{Referencias}

Bedard, Jean C. \& Johnstone, Karla (2004).

Earnings Manipulation Risk, Corporate Governance Risk, and Auditors' Planning and Pricing Decisions. The Accounting Review, 79 (2), 277-304.

Brasil, Comissão de Valores Mobiliários, CVM (2009). Instruction CVM 480, 7 de dezembro de 2009. Disponible en: http://www. cvm.gov.br/ingl/regu/CVMINST_480.asp

Brasil, Comitê de Pronunciamentos Contábeis, Comité de Pronunciamientos Contables, CPC (13, 05 de dezembro de 2008). Adoção inicial da lei $n^{\circ}$ 11.638/07 e medida provisória $n^{\circ}$ 449/08. Brasília - DF. Disponible en: http://www.cpc.org.br/CPC/ Documentos-Emitidos/Pronunciamentos/ Pronunciamento? Id=44

Brasil, Congresso Nacional. Lei 6.404/1976, dispõe sobre as sociedades por ações. Diário Oficial da União, DOU, Poder Executivo, 
Brasília, DF, 17 de dezembro de 1976, suplemento. Disponible en: http://www. planalto.gov.br/ccivil_03/leis/16404consol. htm, http://www.portaldecontabilidade. com.br/legislacao/lei6404_1976.htm

Brasil, Congresso Nacional. Lei 11.638/2007, altera e revoga dispositivos da Lei 6.404, de 15 de dezembro de 1976, e da Lei 6.385, de 7 de dezembro de 1976, e estende às sociedades de grande porte disposições relativas à elaboração e divulgação de demonstrações financeiras. Diário Oficial da União, DOU, edição extra, Poder Executivo, Brasília, DF, 28 de dezembro de 2007. Disponible en: http:/www.planalto.gov.br/ccivil_03/_ ato2007-2010/2007/lei/l11638.htm

Brasil, Conselho Federal de Contabilidade, CFC (2005). Resolução 1034/05. Aprova a NBC P 1.2 - Independência. Disponible en: http://www.portaldecontabilidade. com.br/nbc/nbcp1_2.htm, http://www. portaldecontabilidade.com.br/nbc/ resolucoes.htm

Braunbeck, Guillermo Oscar (2010). Determinantes da qualidade das auditorias independentes no Brasil. São Paulo, Tese de doutorado em Controladoria e Contabilidade, Universidade de São Paulo, São Paulo, Brasil. Disponible en: http://www.teses. usp.br/teses/disponiveis/12/12136/tde04112010-161444/fr.php

DeAngelo, Linda Elizabeth (1981). Auditor Independence, 'Low Balling', and Disclosure Regulation. Journal of Accounting and Economics, 3 (2), 113-127.

Eshleman, John D. \& Guo, Peng (2013). Abnormal Audit Fees and Audit Quality: New
Evidence. American Accounting Association, Annual Meeting, Anaheim, California, August 7, 2013. Disponible en: http://aaahq.org/AM2013/abstract. cfm?submissionID $=688$

Francis, Jere R. (1984). The Effect of Audit Firm Size on Audit Prices: a Study of the Australian Market. Journal of Accounting and Economics, 6 (2), 133-151.

Frankel, Richard M.; Johnson, Marilyn F. \& Nelson, Karen K. (2002). The Relation between Auditors' Fees for Non-Audit Services and Earnings Management. The Accounting Review, 77 (s-1, Supplement), 71-105. Disponible en: http://dspace.mit.edu/bitstream/ handle/1721.1/48912/relationbetweena00fran.pdf?s..

Formigoni, Henrique; Antunes, Maria Thereza Pompa; Leite, Rafael Soãres \& Paulo, Edílson (2008). A contribuição do rodízio de auditoria para a independência e qualidade dos serviços prestados: um estudo exploratório baseado na percepção de gestores de companhias abertas brasileiras. Revista Contabilidade Vista e Revista, Universidade Federal de Minas Gerais, Belo Horizonte, 19 (3), 149-167, Julho/Setembro 2008. Disponible en: http://www.spell.org. br/documentos/ver/8095/a-contribuicaodo-rodizio-de-auditoria-para-a-i---

Gupta, Parveen P.; Krishnan, Gopal V. \& Yu, Wei (2012). Do Auditors Allow Earnings Management When Audit Fees are Low? Disponible en: http://papers.ssrn.com/sol3/papers. cfm?abstract_id=1836829

Hallak, Rodrigo Telles Pires \& Silva, Andre Luiz Carvalhal da (2012). Determinantes 
das despesas com serviços de auditoria e consultoria prestados pelo auditor independente no Brasil. Revista Contabilidade \& Finanças, 23 (60), 223-231. Disponible en: http://www.scielo.br/pdf/rcf/v23n60/ a07v23n60.pdf

Hanlon, Michelle; Krishnan, Gopal V. \& Mills, Lillian F. (2012). Audit Fees and Book-Tax Differences. The Journal of the American Taxation Association, 34 (1), 55-86. Disponible en: http://hdl.handle.net/1721.1/75115

Kinney, William R. \& Libby, Robert (2002).

Discussion of the Relation between Auditors' Fees for Non-Audit Services and Earnings Management. Accounting Review, 77, 107-114. DOI: 10.2308/accr.2002.77.s-1.107

Krishnan, Gopal V. \& Visvanathan, Gnanakumar (2009). Do Auditors Price Audit Committee's Expertise? The Case of Accounting vs. non-Accounting Financial Experts. Journal of Accounting, Auditing \& Finance, 24 (1), 115-144. Disponible en: http://papers.ssrn.com/sol3/papers. cfm?abstract_id=1003686

Larcker, David F. \& Richardson, Scott (2004). Fees Paid to Audit Firms, Accrual Choices, and Corporate Governance. Journal of Accounting Research, 42 (3), 625-658.

Moore, Don A.; Tetlock, Philip E.; Tanlu, Lloyd \& Bazerman, Max H. (2006). Conflicts of Interest and the Case of Auditor Independence: Moral Seduction and Strategic Issue Cycling. Academy of Management Review, 31 (1), 1-20.

Disponible en: http://faculty.haas.berkeley. edu/tetlock/Vita/Philip\%20Tetlock/ Phil\%20Tetlock/2004_Current/2005\%20 Conflicts\%20of\%20interest $\% 20$ and $\% 20$ auditor\%20independencepageproofs.pdf Palmrose, Zoe-Vonna (1986). Audit Fees and Auditor Size: Further Evidence. Journal of Accounting Research, 24 (1), 97-110.

Simunic, Dan A. (1980). The Pricing of Audit Services: Theory and Evidence. Journal of Accounting Research, 18 (1), 161-190. Disponible en: http:// wlkc.gdqy.edu.cn/res/skillsres/ resources/2012/04/05/18/38E1C260-622E4BE0-A260-C2B107218D65.pdf

Watts, Ross L. \& Zimmerman, Jerold L. (1986). Positive Accounting Theory. Englewood Cliffs, New Jersey: Prentice Hall.

- Fecha de recepción: 1 de julio de 2014

- Fecha de aceptación: 21 de octubre de 2014

- Disponible en línea: 15 de diciembre de 2014

\section{Para citar este artículo}

Moraes, Arquimedes de Jesus; Pinheiro, Eduardo José \& Negrelli, Alexsandro Ronchi (2014). Determinantes de costos de auditoría - non audit fee [número especial: Contabilidad Gerencial]. Cuadernos de Contabilidad, 15 (39), 883-895.

doi: 10.11144/Javeriana.cc15-39.dcan 
